\title{
Entanglement enhancement and postselection for two atoms interacting with thermal light
}

\author{
K. Brádler and R. Jáuregui \\ Instituto de Física, Apdo. Postal 20-364, México 01000, Méxicd*
}

(Dated: August 14, 2018)

\begin{abstract}
The evolution of entanglement for two identical two-level atoms coupled to a resonant thermal field is studied for two different families of input states. Entanglement enhancement is predicted for a well defined region of the parameter space of one of these families. The most intriguing result is the possibility of probabilistic production of maximally entangled atomic states even if the input atomic state is factorized and the corresponding output state is separable.
\end{abstract}

PACS numbers: 42.50.Dv, 03.67.-a

Keywords: Atom-light interaction, Entanglement generation

Entanglement of atomic systems is a promising resource for performing various computational and communication tasks originated in quantum information theory. Recent experimental progress has been achieved in the field of creation of multipartite highly nonclassical states in a linear Paul trap [1, 2]. Production of such states requires a nontrivial implementation of a sequence of nonlocal operations (unitary gates) that entangle originally uncorrelated atoms in a desired way. Apart from these sophisticated methods for manipulating the atomic states with the help of a precisely tuned laser field, it has been predicted that originally independent atoms may become entangled after the interaction with a thermal electromagnetic field. At first sight this may seem counterintuitive because thermal light is considered to be a decoherence source. But indeed, if we stay in the quite modest case of entangling just two atoms, Kim et al. [3] as well as Bose et al. [4] and An et al. [5] have reported the occurrence of entanglement of initially separable mixed states after the interaction with thermal light. These studies belong to a broader group of works on the behavior of an atomic ensemble coupled to different kinds of bosonic environment [6, 7].

Loosely speaking, all previous studies of the coupling of an atomic system to a thermal bath [3, 4, [5, 8] concluded with the interesting result that entanglement not only does not disappear after the interaction but it can be even created from originally separable states. In this work we go further. In the framework of the model describing the interaction of an atomic ensemble and a single mode photon field worked out by Tavis et al. [9] we study two physically relevant families of initial pure atomic states coupled to a thermal bath. The states can be tuned by changing its Schmidt number (characterizing the degree of entanglement of initial states) from factorized states to all four maximally entangled states (Bell states). The calculations give quite unexpected results that, to our knowledge, have not been reported so far. It is found that for an input state of the form $\left|\Psi_{\beta}\right\rangle=\sin \beta|00\rangle+\cos \beta|11\rangle$ and for a sufficiently 'hot' environment, there is a stable nonzero probability of producing a maximally entangled state irrespective of the value of $\beta$ and, what is more interesting, despite of the fact that the resulting state after the interaction is separable. This procedure is usually called postselection. Thus we present here a probabilistic source of maximally entangled atomic pairs.

A detailed study of the family of input atomic states $\left|\Phi_{\beta}\right\rangle=\sin \beta|01\rangle+\cos \beta|10\rangle$ shows that entanglement of output states is higher in comparison with input states for a wide interval of $\beta$ values. The picture started in Refs. [3, 6] is now completed by showing that the entangling properties of a thermal field prevail even at very low temperatures, so that vacuum fluctuations can generate partially entangled states from initially factorized states.

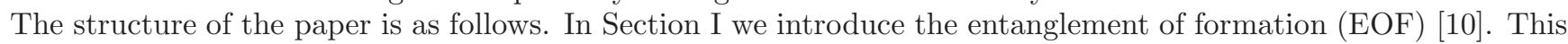
entanglement measure is calculated for general two-qubit states with the help of the analytical expression for the concurrence 11]. In Section $\Pi$ we describe the Tavis-Jaynes-Cummings model setting the stage for the main results presented in two subsections where the evolution of thermal light with two different atomic input configurations $\left|\Phi_{\beta}\right\rangle$ and $\left|\Psi_{\beta}\right\rangle$ is studied. The details about the calculations are given in Appendix.

*Electronic address: kbradler@epot.cz, rocio@fisica.unam.mx 


\section{ENTANGLEMENT MEASURES}

Most axioms required for entanglement measures [12] are accomplished by the entanglement of formation [10] (EOF) defined by

$$
E_{F}(\varrho)=\inf _{\varrho=\sum_{i} p_{i}|\varphi\rangle\left\langle\left.\varphi\right|_{i}\right.} \sum_{i} p_{i} E\left(|\varphi\rangle\left\langle\left.\varphi\right|_{i}\right)\right.
$$

where $E(\bullet)=S\left(\operatorname{Tr}_{1(2)}\left[|\varphi\rangle\left\langle\left.\varphi\right|_{i}\right]\right)\right.$ is called the entropy of entanglement (the von Neumann entropy of the state of interest that is traced over one of its subsystems). The EOF is generally uneasy to calculate even for the lowest dimensional systems. However, Hill and Wootters proved [11] that the EOF can be calculated analytically in the case of an arbitrary two qubit state $\varrho$. They showed that

$$
E_{F}(\varrho)=h\left(\frac{1}{2}\left(1+\sqrt{1-C^{2}(\varrho)}\right)\right)
$$

where $h(x)=-x \log _{2}(x)-(1-x) \log _{2}(1-x)$ is the Shannon entropy and

$$
C(\varrho)=\max \left\{0, \lambda_{1}-\lambda_{2}-\lambda_{3}-\lambda_{4}\right\} .
$$

The parameters $\lambda_{i}$ in Eq. (3) are eigenvalues in descending order of the square root of the matrix

$$
\tilde{R}(\varrho)=\varrho\left(\sigma_{y} \otimes \sigma_{y}\right) \varrho^{*}\left(\sigma_{y} \otimes \sigma_{y}\right)
$$

with $*$ representing the complex conjugation in the standard basis and $\sigma_{y}$ is the $y$-Pauli matrix. $C$ is called the concurrence and since the EOF is monotonous in $C$, the concurrence can also be considered as the entanglement measure. Nevertheless, as emphasized in [13], the proper entanglement measure is the EOF. Using the concurrence for this purpose could cause confusion when investigating entanglement of systems with dimensionality $n \times n$ for $n \neq 2$ since in that case the definition of concurrence is not unique.

Also in some cases, e.g. in [3], the negativity [14] as an entanglement measure is preferred. The reason is that it is easier to calculate (tracing over a partially transposed density matrix). On the other hand, the negativity does not coincide with the entropy of entanglement introduced above for pure bipartite states where it is the unique measure of entanglement [15]. Rather recently [16], connection of the negativity with the concurrence (and thus to the EOF that coincides with the entropy of entanglement for pure bipartite states) was discovered in terms of convex roof construction. Anyhow, in this paper the particular forms of the density matrices allow us to calculate the EOF directly from the concurrence.

The second note concerns the term Schmidt number. It characterizes the degree of entanglement of input states but does not uniquely determine the behavior after the interaction. In other words, even if two input states have the same Schmidt number, for example two Bell states, their evolution and thus entanglement after the interaction may be completely different.

\section{INTERACTION OF TWO ATOMS WITH THERMAL LIGHT}

The Hamiltonian of two identical two-level atoms interacting with a single-mode electromagnetic field in the dipole approximation and standard notation is given by

$$
\begin{aligned}
H & =H_{0}+H_{\text {int }} \\
& =\hbar \Omega\left(b^{\dagger} b+\frac{1}{2}\right)+\frac{\hbar \Omega}{2}\left(\sigma_{z}^{(1)}+\sigma_{z}^{(2)}\right)+\hbar g\left(\sum_{i=1}^{2} \sigma_{+}^{(i)} b+\sigma_{-}^{(i)} b^{\dagger}\right),
\end{aligned}
$$

where resonance of the photon energy and the atomic level splitting is assumed, and the rotating-wave approximation is used. The analogous Hamiltonian for $N$-atoms can be analytically diagonalized as already shown in Ref. [9]. Here we use the dressed states formalism to obtain the eigenvalues and eigenvectors. Since $\left[H_{0}, H_{\text {int }}\right]=0, H_{\text {int }}$ induces transitions only between the degenerate states of $H_{0}$ that, for a given field excitation number $n$, constitutes the tetrad $\{|n\rangle|11\rangle,|n+1\rangle|10\rangle,|n+1\rangle|01\rangle,|n+2\rangle|00\rangle\}_{n}$. The bare states form a 'semilogical' basis where, e.g., $|n+1\rangle|10\rangle$ represents the state with $(n+1)$ photons, the first atom in the excited state (logical state one) and the second one in the ground state (logical state zero). Expressing the interaction Hamiltonian in this basis we get a block diagonal 
matrix with the $n$-th block given by

$$
H_{\text {int }}^{(n)}=\hbar g\left(\begin{array}{cccc}
0 & \sqrt{n+1} & \sqrt{n+1} & 0 \\
\sqrt{n+1} & 0 & 0 & \sqrt{n+2} \\
\sqrt{n+1} & 0 & 0 & \sqrt{n+2} \\
0 & \sqrt{n+2} & \sqrt{n+2} & 0
\end{array}\right) .
$$

After normalization of the eigenvectors of this matrix, the dressed basis states read

$$
\begin{aligned}
|1\rangle_{n} & =\sqrt{\frac{n+1}{2 n+3}}\left[-\sqrt{\frac{n+2}{n+1}}|n\rangle|11\rangle+|n+2\rangle|00\rangle\right] \\
|2\rangle_{n} & =\sqrt{\frac{1}{2}}[|n+1\rangle|10\rangle-|n+1\rangle|01\rangle] \\
|3\rangle_{n} & =\sqrt{\frac{2}{4 n+6}}\left[\sqrt{\frac{n+2}{n+1}}|n\rangle|11\rangle-\sqrt{\frac{2 n+3}{2 n+4}}|n+1\rangle|10\rangle-\sqrt{\frac{2 n+3}{2 n+4}}|n+1\rangle|01\rangle+|n+2\rangle|00\rangle\right] \\
|4\rangle_{n} & =\sqrt{\frac{n+2}{4 n+6}}\left[\sqrt{\frac{n+2}{n+1}}|n\rangle|11\rangle+\sqrt{\frac{2 n+3}{2 n+4}}|n+1\rangle|10\rangle+\sqrt{\frac{2 n+3}{2 n+4}}|n+1\rangle|01\rangle+|n+2\rangle|00\rangle\right] .
\end{aligned}
$$

In particular, the atomic state $1 / \sqrt{2}(|10\rangle-|01\rangle)$ is a dark state.

\section{A. Initial Atomic State $\left|\Phi_{\beta}\right\rangle$}

In this section, the system light-atoms is assumed to be initially in a factorized state $\varrho^{(\Phi)}(\beta, 0)=\varrho_{\text {light }} \otimes \varrho_{\text {atoms }}^{(\Phi)}$ where the atomic state is a pure state

$$
\left|\Phi_{\beta}\right\rangle=\sin \beta|01\rangle+\cos \beta|10\rangle
$$

with the Schmidt number $\sin \beta$ and the light considered to be in a thermal state

$$
\begin{aligned}
\varrho_{\text {light }} & =\left[1-e^{-\hbar \Omega / k T}\right] e^{\hbar \Omega / 2 k T} \sum_{n=0}^{\infty} e^{-(n+1 / 2) \hbar \Omega / k T}|n\rangle\langle n| \\
& =\sum_{n=0}^{\infty} \frac{e^{\bar{n}} \bar{n}^{n}}{n !}|n\rangle\langle n|,
\end{aligned}
$$

with $\bar{n}=\left[e^{\hbar \Omega / k T}-1\right]^{-1}$ the average number of photons. After a direct but otherwise tedious calculation the time evolved density matrix $\varrho^{(\Phi)}(\beta, t)$ can be obtained. Since we are interested in the entanglement behavior of the atomatom system, we trace over the electromagnetic field states. The resulting time-dependent reduced density matrix is of the form

$$
\tilde{\varrho}^{(\Phi)}(\beta, t)=\left(\begin{array}{cccc}
a_{1} & 0 & 0 & 0 \\
0 & a_{2} & a_{3} & 0 \\
0 & a_{3} & a_{4} & 0 \\
0 & 0 & 0 & a_{5}
\end{array}\right) .
$$

The specific expressions of the matrix elements can be found in Appendix.

The structure of $\tilde{\varrho}^{(\Phi)}$ is very friendly for the calculation of the concurrence with Eq. (3) and consequently the EOF. The result is illustrated in Fig. 1 where oscillations of the EOF are noticeable for all $\beta$. There is a $\beta_{0} \ll 1$ that depends on $\bar{n}$ such that for the subinterval $\beta_{0}<\beta<\pi / 2-\beta_{0}$ the minimum value of the EOF is zero and the local maxima (revivals) are always smaller than the initial value for $\bar{n}>0$ and equal for $\bar{n}=0$. Thus, the EOF of the atomic entangled states is reduced in the presence of thermal light. As the temperature increases the temporal width of the revivals decreases. A brief inspection of the picture reveals other interesting aspects of interacting light with this family of states $\left|\Phi_{\beta}\right\rangle$ in the second interval of $\beta, \pi / 2<\beta<\pi$. Due to the fact that $1 / \sqrt{2}(|10\rangle-|01\rangle)$ is a dark state the behavior is completely different. The EOF exhibits very soft oscillations and is never smaller than its initial value. We thus see that in this region, the resulting state is stable under the interaction with thermal light. This could be an important hint for quantum engineers (and protocol designers generally) telling them that there 


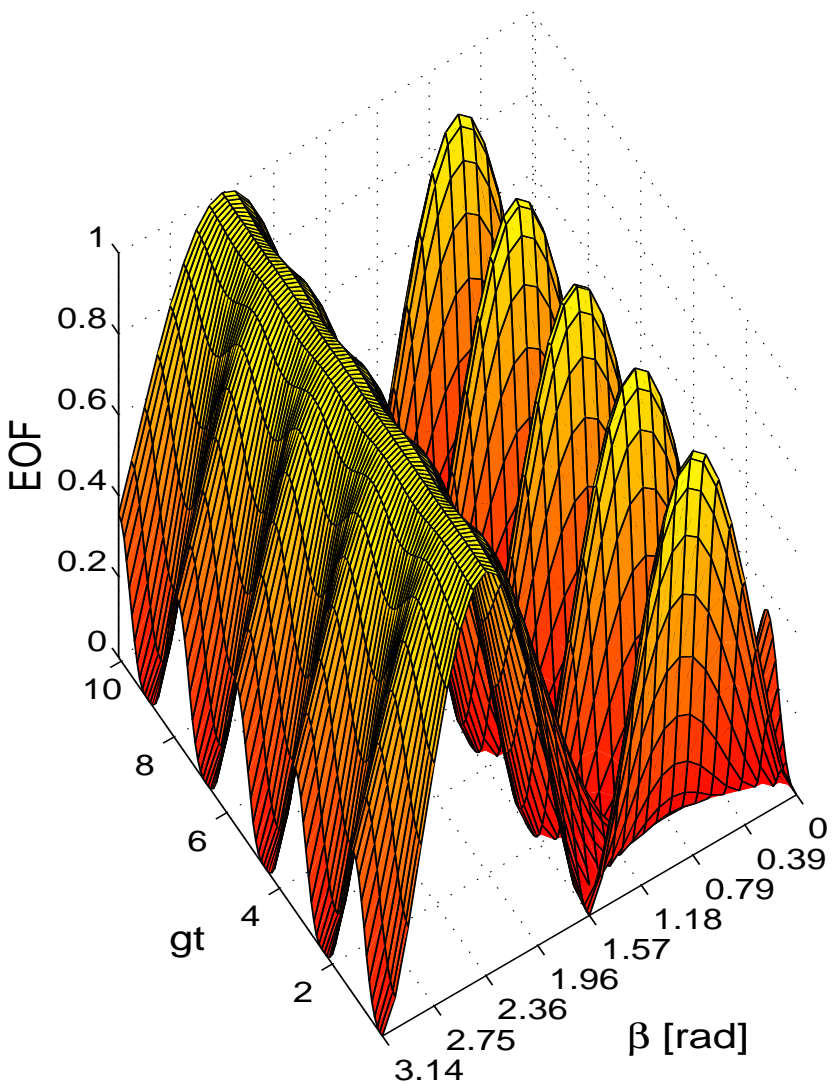

(a)

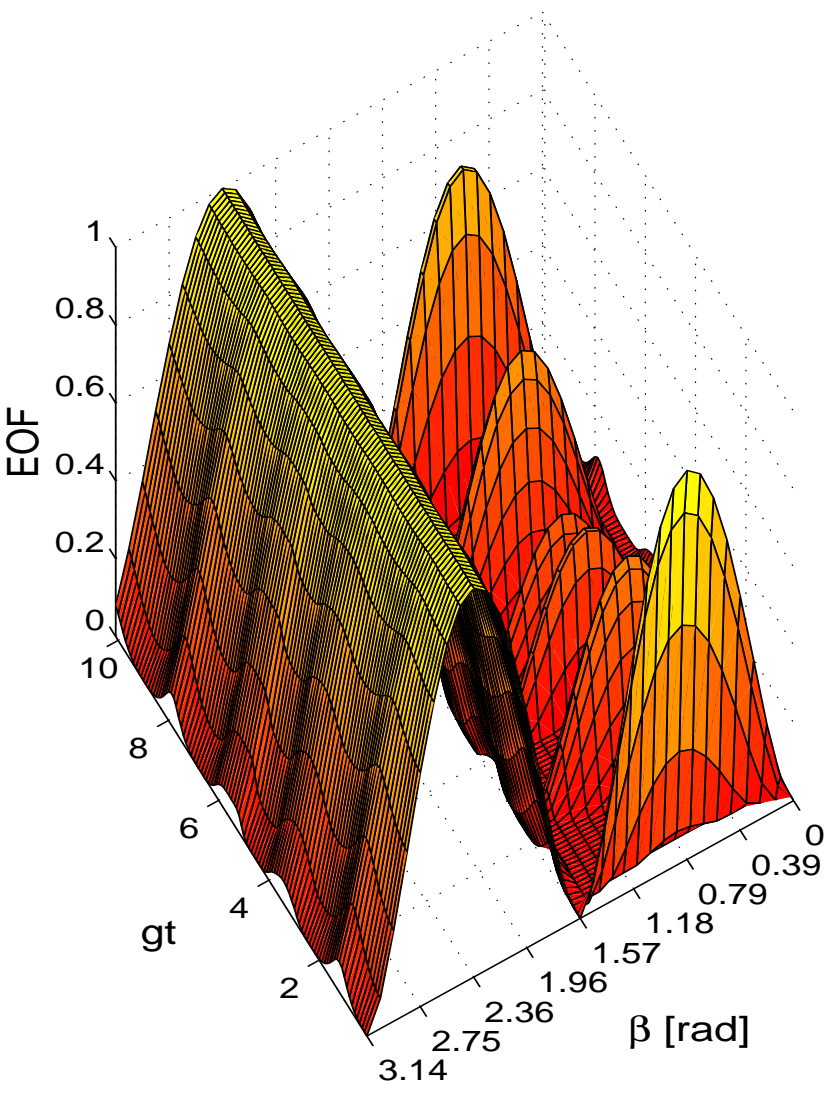

(b)

FIG. 1: The EOF of the output state given by Eq. (10) for interacting atomic states $\left|\Phi_{\beta}\right\rangle$ with: (a) a zero photon field, and (b) a thermal field with a nonzero average photon number $(\bar{n} \approx 0.64)$. A completely different behavior for $0 \leq \beta \leq \pi / 2$ and $\pi / 2 \leq \beta \leq \pi$ can be seen. EOF revivals are observed in the first interval where, generally, entanglement of input states is devalued except for initially factorized or weakly entangled states. On the other hand, in the second interval the EOF is never lower than its initial value. We can observe significant values of the EOF for initially factorized atomic states and $\bar{n}=0$.

exists an important class of initial atomic states where the interaction with a thermal field means not only no loss of entanglement but even its enhancement without strong fluctuations. The works presented so far on this topic (e.g. Refs. [3, 4] ) were limited only to input atomic states with small values of the Schmidt number $0<\beta<\beta_{0} \ll 1$ and recognized the presence of the dark state but do not discuss the stability of entanglement in the whole range of $\beta$.

For $\bar{n}=0$ the matrix elements of Eq. (10) have a particulary simple form

$$
\begin{aligned}
& a_{1}=\frac{1}{4}(1+\sin 2 \beta)\left(1-\cos 2 \alpha_{-1} t\right) \\
& a_{2}=\frac{1}{8}(1+\sin 2 \beta)\left(1+\cos 2 \alpha_{-1} t\right)-\cos 2 \beta \cos \alpha_{-1} t+\frac{1}{4}(1-\sin 2 \beta) \\
& a_{3}=\frac{1}{8}(1+\sin 2 \beta)\left(1+\cos 2 \alpha_{-1} t\right)-\frac{1}{4}(1-\sin 2 \beta) \\
& a_{4}=\frac{1}{8}(1+\sin 2 \beta)\left(1+\cos 2 \alpha_{-1} t\right)+\cos 2 \beta \cos \alpha_{-1} t+\frac{1}{4}(1-\sin 2 \beta) \\
& a_{5}=0 .
\end{aligned}
$$

By examining the density matrix for $\beta=0$ we may exactly see the influence of the vacuum fluctuations on a factorized input state. We see that there is a $50 \%$ probability of finding the system with both atoms in their ground state and a single photon, while there is a $50 \%$ probability that this photon has been reabsorbed by one of the atoms leaving the atomic system in the entangled state. As a consequence, spontaneous decay may be regarded as a causative mechanism for inducing entanglement between the two two-level atoms when their initial state $\left|\Phi_{\beta}\right\rangle$ is practically unentangled $(\beta \simeq 0)$ or to enhance it for different $\beta$ in the interval $\pi / 2 \leq \beta \leq \pi$. 

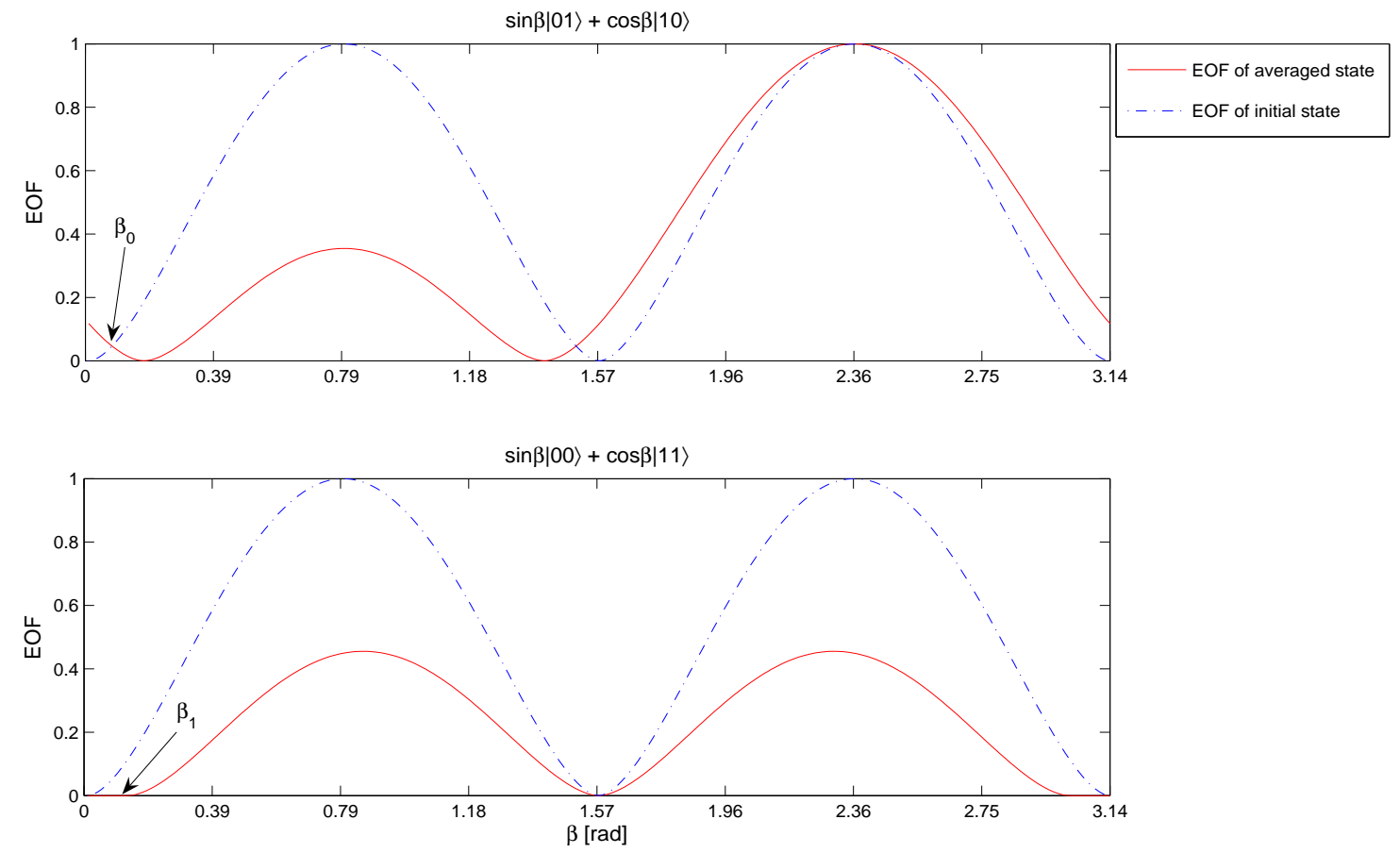

FIG. 2: The continuous lines in both plots are the EOF for mixtures of the output states occurring within the time interval where the EOF is periodic $(\bar{n}=0)$. The dash-dotted lines correspond to the EOF for $t=0$ (initial states). In the upper plot the interval $\beta_{0}<\beta<\pi / 2-\beta_{0}$ with $\beta_{0}=1 / 2 \arcsin (1 / 7)$ demarcates the region with lower entanglement in comparison to corresponding input states. In the bottom plot we can see that entanglement is not present in the interval $\left(-\beta_{1}, \beta_{1}\right)$, with $\beta_{1}=\arctan (1 / 8)$, hence disappearing even for initially weakly entangled states.

The fact that EOF exhibits strong oscillations as already illustrated may limit even its evaluation. Evaluation of the entanglement requires knowledge of the matrix elements and these cannot be determined just from one copy of an unknown quantum state. One possibility is to have many copies of the same unknown state and perform quantum state tomography. Recent findings show that the tomography is not necessary [17, 18] but still does hold that it is not possible to measure the entanglement from just one copy of a state. Besides, if the average number of photons is so large that the time window for picking the desired state is too long compared to the length of the oscillations, the measurement could be interpreted as a random pick from the set of all possible outputs and a proper time average must be performed.

Let us study this question for the electromagnetic field initially in the vacuum state as an illustration. In this simple case, the natural period is determined by the commeasurable Rabi frequencies $\sqrt{8} g$ and $\sqrt{2} g$

$$
\tilde{\varrho}^{(\Phi)}(\beta)=\frac{1}{\sqrt{2} \pi} \int_{0}^{\sqrt{2} \pi} \varrho(\beta, t) \mathrm{d}(g t)=\frac{1}{8}\left(\begin{array}{cccc}
2(1+\sin 2 \beta) & 0 & 0 & 0 \\
0 & 3-\sin 2 \beta & 3 \sin 2 \beta-1 & 0 \\
0 & 3 \sin 2 \beta-1 & 3-\sin 2 \beta & 0 \\
0 & 0 & 0 & 0
\end{array}\right) .
$$

The resulting EOF can be seen in the upper plot of Fig. 2 and, we are able to confirm the qualitative conclusions about the entanglement behavior based on Fig. 1 for the averaged EOF. As mentioned above, we can observe nonzero entanglement for initially factorized states. For entangled states with $\beta_{0}<\beta<\pi / 2-\beta_{0}$ the averaged EOF is lower than for the input states. In general, for the remaining $\beta$ the EOF average is higher than the initial value of the EOF.

The extension of this averaging process for the case of an interaction with thermal light with $\bar{n}>0$ requires the identification of a natural time scale since in this case an infinite number of the incommensurable Rabi frequencies $\alpha_{n-1}=2 g \sqrt{n+1 / 2}$ determines the evolution of the system. One such scale could be found by the following procedure. Writing the summations in the expression of $\tilde{\varrho}^{(\Phi)}(\beta, t)$ in an integral form using the Abel-Plana formula, it can be shown that the adimensional parameter $g t \sqrt{2 k T / \hbar \Omega}$ is especially important to understand the evolution of the system. In fact, it can be used to define a natural time $\tau_{0}=g^{-1} \sqrt{\hbar \Omega / 2 k T}$ to distinguish short and long time effects of the interaction (see Appendix for more details). If the average procedure from above is applied, $\tau_{0}$ could also be used for this purpose. 


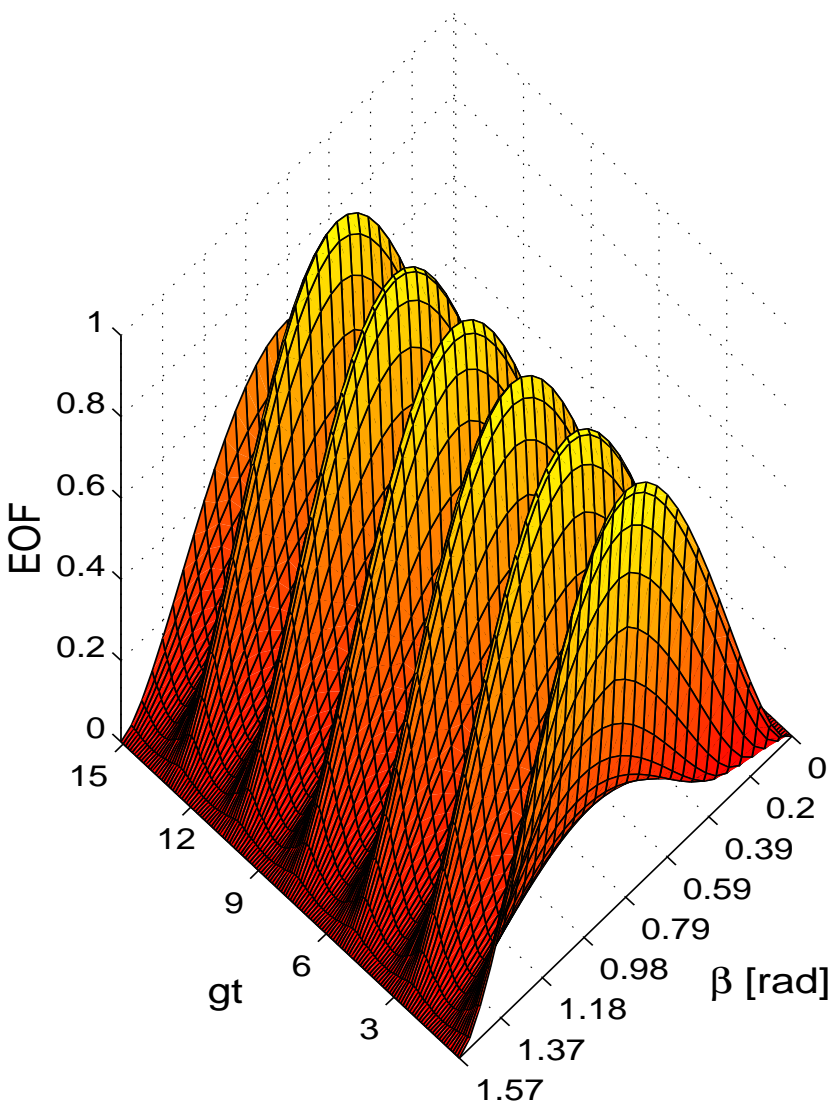

(a)

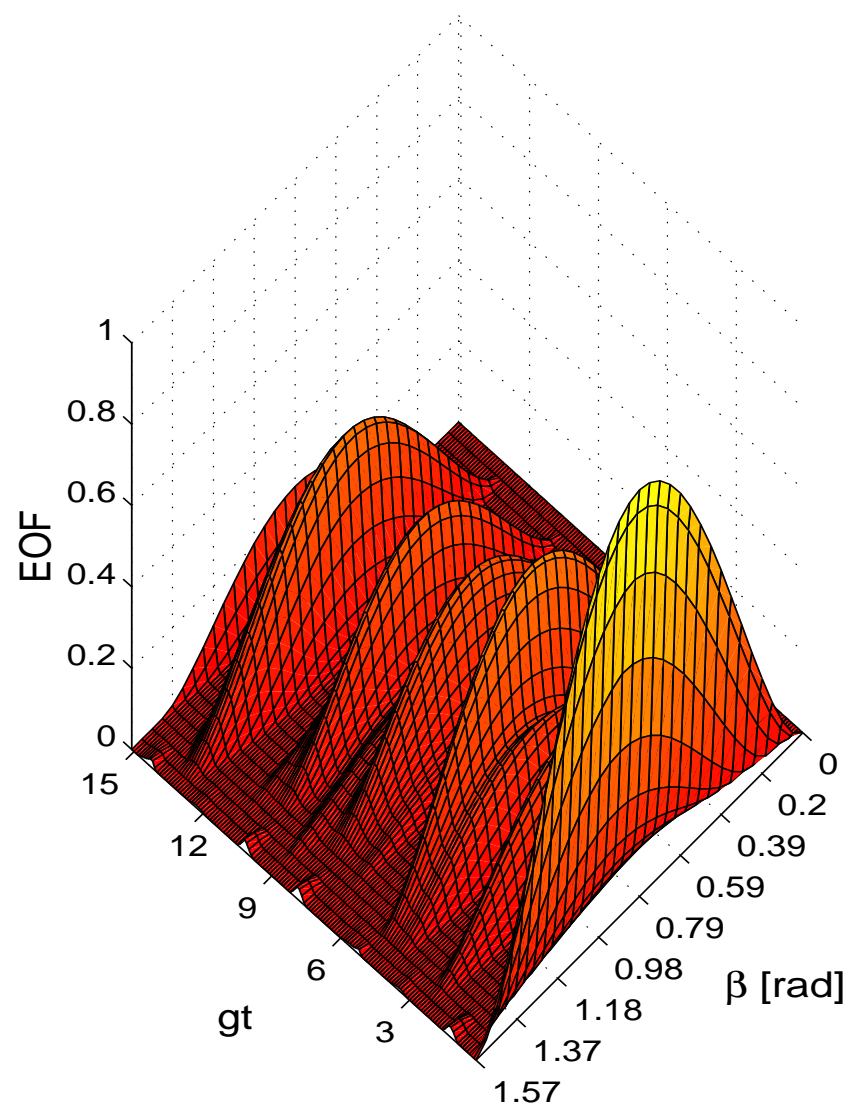

(b)

FIG. 3: The EOF of output state (14) for interacting atomic states $\left|\Psi_{\beta}\right\rangle$ with (a) a zero photon field, (b) a thermal field with $\bar{n} \approx 0.64$. Notice the small enhancement of the EOF for $\beta \sim \pi / 2$ where $\bar{n}$ was chosen to provide a maximum value of the enhancement.

\section{B. Initial Atomic State $\left|\Psi_{\beta}\right\rangle$}

Following the same reasoning as in the previous subsection, but for an initial state of the form

$$
\left|\Psi_{\beta}\right\rangle=\sin \beta|00\rangle+\cos \beta|11\rangle
$$

interacting with light in a thermal state (9), we arrive to a reduced density matrix with the structure

$$
\tilde{\varrho}^{(\Psi)}(\beta, t)=\left(\begin{array}{cccc}
b_{1} & 0 & 0 & b_{2} \\
0 & b_{3} & b_{3} & 0 \\
0 & b_{3} & b_{3} & 0 \\
b_{2}^{*} & 0 & 0 & b_{4}
\end{array}\right) .
$$

The particular expressions for the matrix elements are given in Appendix. As illustrated in Fig. 3 , in this case a resonant thermal electromagnetic field is not able to induce, in general, atomic entanglement for input factorized atomic states, nor to enhance it for initial already entangled states. An exception is provided for the states with $\beta \simeq \pi / 2$. There, a very slight EOF enhancement is observed for $0<\bar{n} \lesssim 2$. This effect is discussed at length in Ref. [3] and present, but barely observable, in Fig. 3. For the other values of $\beta$, at most, partially entangled input states exhibit revivals of the EOF. Thus, in comparison to the input state $\left|\Phi_{\beta}\right\rangle$, the entanglement properties of a thermal field are not so striking. This qualitative observation is confirmed for $\bar{n}=0$ by the mixture calculation in the spirit of the previous section. It gives us

$$
\tilde{\varrho}^{(\Psi)}(\beta)=\frac{1}{12}\left(\begin{array}{cccc}
4(2-\cos 2 \beta) & 0 & 0 & 4 \sin 2 \beta \\
0 & \cos ^{2} \beta & \cos ^{2} \beta & 0 \\
0 & \cos ^{2} \beta & \cos ^{2} \beta & 0 \\
4 \sin 2 \beta & 0 & 0 & 6 \cos ^{2} \beta
\end{array}\right)
$$




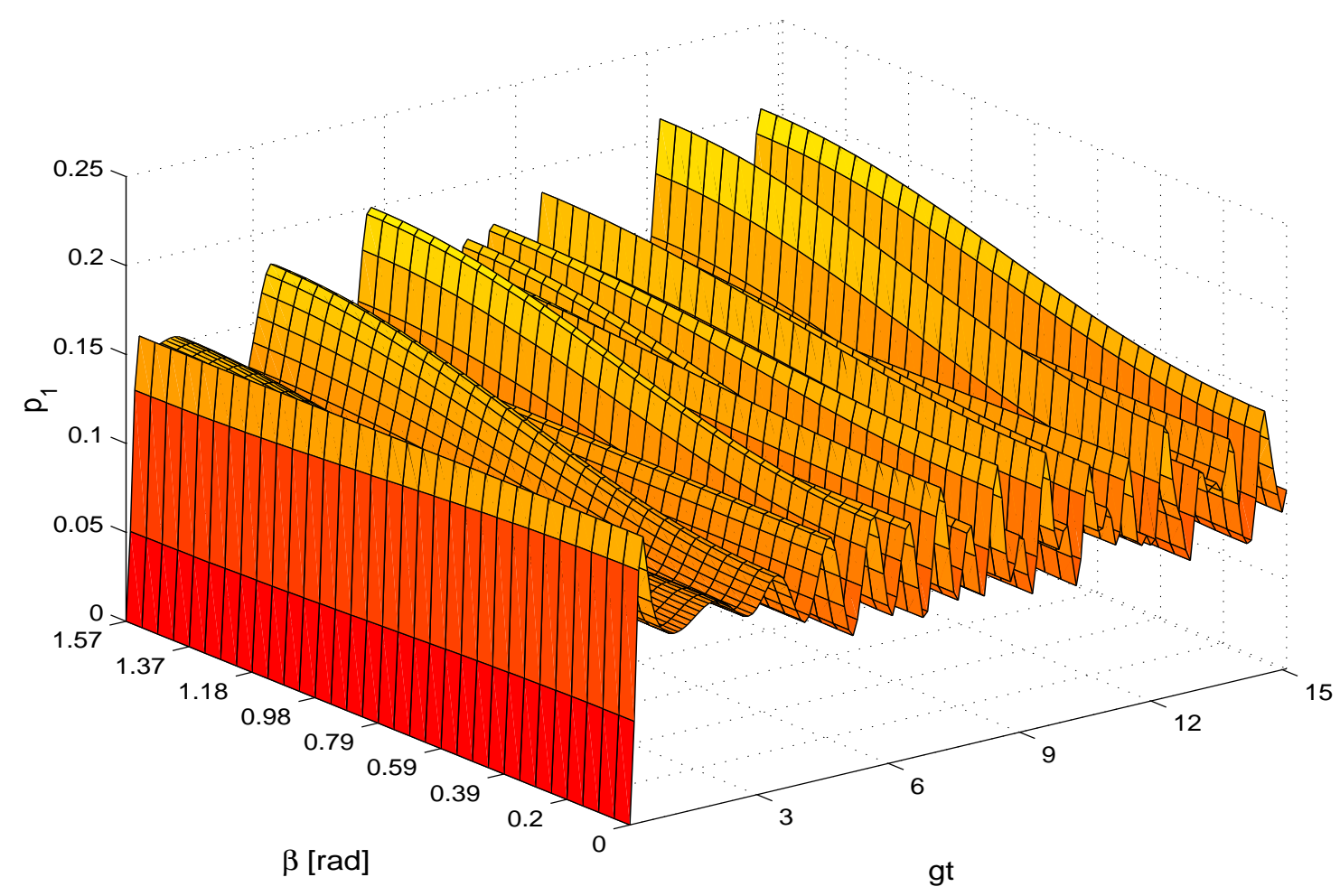

FIG. 4: Depicted values of parameter $p_{1}$ for $\bar{n} \approx 5.9$ which determines the probability of the successful postselection of a maximally entangled state $1 / \sqrt{2}(|10\rangle+|01\rangle)$ from outgoing state (14). It is possible to postselect the state irrespective of $\beta$ and thus it can be done even if the output state is separable.

The resulting EOF is depicted in Fig. 2 (bottom plot). We see that in the mixture sense, there is not only a separable output for initially unentangled states, as discussed above, but also for some of the input partially entangled states. Also, if we compare the EOF for the initial state (dash dotted line) and evolved state (continuous line) the output states are much less entangled in general.

Nevertheless, returning to the evolved state (14), another interesting aspect appears. Eq. (14) can be written as

$$
\tilde{\varrho}^{(\Psi)}(\beta, t)=p_{1} \varrho_{1}+\left(1-p_{1}\right) \varrho_{2}
$$

where $p_{1} \equiv b_{3}$ and

$$
\begin{aligned}
& \varrho_{1}=\frac{1}{2}|01+10\rangle\langle 01+10| \\
& \varrho_{2}=\frac{1}{b_{1}+b_{4}}\left(b_{1}|00\rangle\left\langle 00\left|+b_{4}\right| 11\right\rangle\left\langle 11\left|+b_{2}\right| 00\right\rangle\left\langle 11\left|+b_{2}^{*}\right| 11\right\rangle\langle 00|\right)
\end{aligned}
$$

and both states can be unambiguously discriminated. To show this, let us consider a projectively measuring apparatus with three possible outputs. Two of them project into orthogonal subspaces spanned by $|00\rangle\langle 00|$ and $|11\rangle\langle 11|$ and the third one is the complement. Then, from the form of (16) it follows that if we do not detect anything on the first and the second output the state is in the required maximally entangled state $\frac{1}{\sqrt{2}}|01+10\rangle$. The probability of this procedure is given by the parameter $p_{1}$. Thus, no additional quantum resource for the discrimination is needed [19].

Therefore, even if the EOF is zero (i.e. when an output state is separable) the entangled pair can be extracted in terms of postselection. Also, it is interesting that the maximum value of $p_{1}$ is almost the same not only for different input states (see Fig. 4) but also for different average photon numbers of thermal light. It is worth remarking that the higher photon average number is used the less fluctuating the parameter $p_{1}$ we get. This constant character of $p_{1}$ is broken only for the case of a zero photon field where, not surprisingly, for an input state $|00\rangle$ we cannot extract a maximally entangled state by any means, see Eq. (15).

Note that a similar procedure cannot be applied to the resulting state in Eq. (10) from the previous subsection. There, we are not able to find a decomposition of a density matrix in the form $\varrho=\varrho_{1} \oplus \varrho_{2}$ as in the current case, which ensures that if two arbitrary density matrices are positive the third must be so. 


\section{CONCLUSIONS}

In this work, we have explored the possibility of preserving or enhancing entanglement between two atoms after their interaction with a thermal field. We have investigated the interaction for two kinds of initial atomic states $\left|\Phi_{\beta}\right\rangle=1 / \sqrt{2}(\sin \beta|01\rangle+\cos \beta|10\rangle)$ and $\left|\Psi_{\beta}\right\rangle=1 / \sqrt{2}(\sin \beta|00\rangle+\cos \beta|11\rangle)$. Although this physical system had been discussed previously, new interesting phenomena have been found and the already reported results have been confirmed. For the initial state $\left|\Phi_{\beta}\right\rangle$, we observe a very different behavior after the interaction with the thermal field for two distinguished intervals of input states. Qualitatively, for almost the whole range $\beta=(0, \pi / 2)$, the EOF is decreased while for the remaining input states there are oscillations in the EOF so that they are never smaller than their initial value. This result is more significant at low temperatures, hence, the entanglement enhancement mechanism is induced by spontaneous decay.

For the second family of input states parameterized by $\left|\Psi_{\beta}\right\rangle$, there is no entanglement enhancement for almost any $\beta$. In this case, the entanglement is generally degraded matching our intuitive conception of an interaction between a correlated pairs of qubits and thermal light. Nevertheless, the output atomic density matrix can be decomposed into $|\phi\rangle=1 / \sqrt{2}|01+10\rangle$ and a partially entangled mixed state, both of them living in mutually orthogonal subspaces. Thus they can be discriminated with zero probability of error. If we consider that this holds for all $\beta$ when $\bar{n}$ is sufficiently high, it follows that we are able to extract the maximally entangled state $|\phi\rangle$ even if the input is a factorized state and the output is in a separable form. We may conclude that we have at disposal a probabilistic source of maximally entangled atomic pairs, when our deposit is just a sufficiently hot thermal light with two atoms in a factorized state.

In current experiments with trapped ions, the energies involved in the transitions compared with room temperatures are such that $\hbar \Omega / k T \ll 1$ for the Innsbruck group $\left(729 \mathrm{~nm}\right.$ atomic transition in $\left.{ }^{40} \mathrm{Ca}^{+}\right)[2]$ and $\hbar \Omega / k T \gg 1$ for the NIST group $\left({ }^{9} \mathrm{Be}^{+}\right.$at $\left.1.2 \mathrm{GHz}\right)$ [1]. Thus, one would expect that the former is a good scenario for testing the predictions made for the initial state $\left|\Phi_{\beta}\right\rangle$, in particular, the entanglement enhancement. Meanwhile, the NIST group parameters could be appropriate to study the possibility of stabilized postselection of the maximally entangled state described for the interaction of thermal resonant light with $\left|\Psi_{\beta}\right\rangle$.

\section{Acknowledgments}

The authors are grateful to S. Hacyan for valuable comments. Funding from CONACyT 41048-F is acknowledged.

\section{APPENDIX}

\section{Initial Atomic State $\left|\Phi_{\beta}\right\rangle$}

With the help of the inverse transformation from the bare state to the dressed state basis, we rewrite the initial density matrix determined by the atomic state $\left|\Phi_{\beta}\right\rangle$ and a thermal single-mode field

$$
\begin{aligned}
\varrho^{(\Phi)}(\beta, t=0)= & \sum_{n=0}^{\infty} \frac{p_{n}}{4}\left[( 1 + \operatorname { s i n } 2 \beta ) ( - | 3 \rangle _ { n } + | 4 \rangle _ { n } ) \left(-\left\langle\left. 3\right|_{n}+\left\langle\left. 4\right|_{n}\right)\right.\right.\right. \\
& -\sqrt{2} \cos 2 \beta\left(( - | 3 \rangle _ { n } + | 4 \rangle _ { n } ) \langle 2 | _ { n } + | 2 \rangle _ { n } \left(-\left\langle\left. 3\right|_{n}+\left\langle\left. 4\right|_{n}\right)\right)-2(1-\sin 2 \beta)|2\rangle\left\langle\left. 2\right|_{n}\right]\right.\right.
\end{aligned}
$$

where $p_{n}=[1-\exp (-\hbar \Omega / k T)] \exp (-n \hbar \Omega / k T)$. The time evolution of the whole system is directly found. Tracing over the resulting density matrix we obtain an output atomic state (Eqs. (10)) with elements

$$
\begin{aligned}
& a_{1}=\frac{1}{8}(1+\sin 2 \beta)\left(m_{+}-h_{2}(2 t)-\frac{1}{2} h_{1}(2 t)\right) \\
& a_{2}=\frac{1}{8}(1+\sin 2 \beta)\left(1+h_{2}(2 t)\right)-\frac{1}{2} h_{2}(t) \cos 2 \beta+\frac{1}{4}(1-\sin 2 \beta) \\
& a_{3}=\frac{1}{8}(1+\sin 2 \beta)\left(1+h_{2}(2 t)\right)-\frac{1}{4}(1-\sin 2 \beta) \\
& a_{4}=\frac{1}{8}(1+\sin 2 \beta)\left(1+h_{2}(2 t)\right)+\frac{1}{2} h_{2}(t) \cos 2 \beta+\frac{1}{4}(1-\sin 2 \beta) \\
& a_{5}=\frac{1}{8}(1+\sin 2 \beta)\left(m_{-}-h_{2}(2 t)+\frac{1}{2} h_{1}(2 t)\right)
\end{aligned}
$$


with

$$
\begin{aligned}
& h_{1}(t)=\sum_{n=0}^{\infty} \frac{p_{n}}{n+1 / 2} \cos \alpha_{n-1} t \\
& h_{2}(t)=\sum_{n=0}^{\infty} p_{n} \cos \alpha_{n-1} t=-\frac{1}{4 g^{2}} h_{1}^{\prime \prime}(t) .
\end{aligned}
$$

$\alpha_{n-1}=2 g \sqrt{n+1 / 2}$ is the Rabi frequency coming from eigenvalues of the interaction Hamiltonian (6) and $m_{ \pm}=$ $1 \pm M \arctan (\exp (-\hbar \Omega / 2 k T))$ where $M=[1-\exp (-\hbar \Omega / k T)] \exp (\hbar \Omega / 2 k T)$.

Expressions (A.7) appearing in the matrix elements can be written in a more compact form

$$
h_{2}(t)=2 \sinh \kappa \sum_{n=0}^{\infty} e^{\kappa(2 n+1)} \cos \sqrt{2 n+1} g t=2 \sinh 2 \kappa \sum_{n=0}^{\infty} e^{\kappa n} \cos \sqrt{n} g t-\sum_{n=0}^{\infty} e^{2 \kappa n} \cos \sqrt{2 n} g t
$$

where $\kappa=\frac{\hbar \Omega}{2 k T}$. Using the Abel-Plana formula

$$
\sum_{n=0}^{\infty} F(n)=\int_{0}^{\infty} F(x) \mathrm{d} x+\frac{1}{2} F(0)+i \int_{0}^{\infty} \frac{F(i x)-F(-i x)}{e^{2 \pi x}-1} \mathrm{~d} x
$$

the following integral expression for $h_{2}(t)$ is found

$$
h_{2}(t)=2 \frac{\sinh \kappa}{\kappa}\left[\int_{0}^{\infty} e^{-x} \cos \tilde{t} \sqrt{x} \mathrm{~d} x+4 \int_{0}^{\infty} \frac{\sin x \cosh \tilde{t} \sqrt{x}-\sin 2 x \cosh \tilde{t} \sqrt{2 x}}{e^{\frac{\pi x}{\kappa}}-1} \mathrm{~d} x\right]
$$

with an adimensional variable $\tilde{t}=\frac{g t}{\sqrt{\kappa}}$ that establishes a natural time scale $\tau_{0}=\frac{\sqrt{\kappa}}{g}$ for the description of the interaction. Notice that the second integral is an exponential decreasing function of the temperature so that for a sufficiently hot environment

$$
h_{2}(t) \sim 2 \frac{\sinh \kappa}{\kappa} \int_{0}^{\infty} e^{-x} \cos \tilde{t} \sqrt{x} \mathrm{~d} x=2 \frac{\sinh \kappa}{\kappa}\left[1-\tilde{t} e^{-\tilde{t}^{2}} \int_{0}^{\tilde{t}} e^{x^{2}} \mathrm{~d} x\right] .
$$

The latter integral can be recognized as an error function with an imaginary argument. An integral expression for $h_{1}(t)$ can be found in a similar manner.

\section{Initial Atomic State $\left|\Psi_{\beta}\right\rangle$}

Calculations similar to the case of the initial state $\left|\Phi_{\beta}\right\rangle$ lead to the following matrix coefficients of Eq. (14)

$$
\begin{aligned}
& b_{1}=\sum_{n=0}^{\infty} p_{n}\left[\sin ^{2} \beta\left(\frac{n-1}{2 n-1}+\frac{n}{2 n-1} \cos \alpha_{n-2} t\right)^{2}+\cos ^{2} \beta \frac{(n+1)(n+2)}{(2 n+3)^{2}}\left(1-\cos \alpha_{n} t\right)^{2}\right] \\
& b_{2}=e^{2 i \Omega t} \sin \beta \cos \beta \sum_{n=0}^{\infty} p_{n}\left(\frac{n-1}{2 n-1}+\frac{n}{2 n-1} \cos \alpha_{n-2} t\right)\left(\frac{n+2}{2 n+3}+\frac{n+1}{2 n+3} \cos \alpha_{n} t\right) \\
& b_{3}=\sum_{n=0}^{\infty} p_{n}\left[\sin ^{2} \beta \frac{n}{4 n-2} \sin ^{2} \alpha_{n-2} t+\cos ^{2} \beta \frac{n+1}{4 n+6} \sin ^{2} \alpha_{n} t\right] \\
& b_{4}=\sum_{n=0}^{\infty} p_{n}\left[\sin ^{2} \beta \frac{n(n-1)}{(2 n-1)^{2}}\left(1-\cos \alpha_{n-2} t\right)^{2}+\cos ^{2} \beta\left(\frac{n+2}{2 n+3}+\frac{n+1}{2 n+3} \cos \alpha_{n} t\right)^{2}\right] .
\end{aligned}
$$

[1] Leibried D, Knill E, Seidelin S, Britton J, Blakestad R B, Chiaverini J, Hume D B, Itano W M, Jost J D, Langer C, Ozeri R, Reichle R, and Wineland D J 2005 Nature 438, 639 
[2] Häfner H, Hänsel W, Roos C F, Benhelm J, Chek-al-kar D, Chwalla M, Körber T, Rapol U D, Riebe M, Schmidt P O , Becher C, Gühne O, Dür W, and Blatt R 2005 Nature 438, 643

[3] Kim M S, Lee J, Ahn D, and Knight P L 2002 Phys. Rev. A 65, 040101

[4] Bose S, Fuentes-Guridi I, Knight P L, and Vedral V 2001 Phys. Rev. Lett. 87, 050401

[5] An J-H, Wang S-J, and Luo H-G 2005 J. Phys. A 38, 3579

[6] Sainz I, Klimov A B, and Roa L 2006 Phys. Rev. A 73, 032303; Braun D 2002 Phys. Rev. Lett. 89, 277901; Kudryavtsev I K, Lambrecht A, Moya-Cessa H and Knight P L 1993 J. Mod. Optics 40, 1605; Tessier T E, Deutsch I H, Delgado A and Fuentes-Guridi I 2003 Phys. Rev. A 68, 062316

[7] Plenio M B and Huelga S F 2002 Phys. Rev. Lett. 88, 197901

[8] Schneider S and Milburn G J 2002 Phys. Rev. A 65, 042107; Lucamarini M, Paganelli S, and Mancini S 2004 Phys. Rev. A 69, 062308

[9] Tavis M and Cummings F W 1967 Phys. Rev. 170, 379

[10] Bennett C H, DiVincenzo D P, Smolin J A, and Wootters W K 1996 Phys. Rev. A 54, 3824

[11] Hill S and Wootters W K 1997 Phys. Rev. Lett. 78, 5022; Wootters W K 1998 Phys. Rev. Lett. 80, 2245

[12] Horodecki M, Horodecki P, and Horodecki R 2000 Phys. Rev. Lett. 84, 2014; Vedral V, Plenio M B, Rippin M A , and Knight P L 1997 Phys. Rev. Lett. 78, 2275

[13] Plenio M B and Virmani S, quant-ph/0504163

[14] Życzkowski K, Horodecki P, Sanpera A, and Lewenstein M 1998 Phys. Rev. A 58, 883; Vidal G and Werner R F 2002 Phys. Rev. A 65, 032314

[15] Popescu S and Rohrlich D 1997 Phys. Rev. A 56, R3319; Donald M J, Horodecki M, and Rudolph O 2002 J. Math. Phys. 43, 4252

[16] Lee S, Chi D P, Oh S D, and Kim J 2003 Phys. Rev. A 68, 062304

[17] Acín A, Tarrach R, and Vidal G 2000 Phys. Rev. A 61, 062307

[18] Horodecki P 2003 Phys. Rev. Lett. 90, 167901

[19] If, from some reasons, we need to preserve both output states $\varrho_{1}, \varrho_{2}$ intact we introduce an auxiliary qubit $|0\rangle_{\text {aux }}$ and the unitary matrix $T=C N O T_{13} C N O T_{23}$ where in $C N O T_{m n} m$ and $n$ is a control and a target qubit, respectively. This is useful for non-demolition discrimination between $\varrho_{1}$ and $\varrho_{2}$ since under this transformation we get $\varrho$ 品 $\otimes|0\rangle\langle 0|$ aux $\stackrel{T}{\rightarrow}$ $p_{1} \varrho_{1} \otimes|1\rangle\left\langle\left. 1\right|_{\text {aux }}+\left(1-p_{1}\right) \varrho_{2} \otimes \mid 0\right\rangle\left\langle\left. 0\right|_{\text {aux }}\right.$. So that, by a projective measurement on the ancilla, $\varrho_{1}$ and $\varrho_{2}$ can be unambigously discriminated without destroying them. 